\title{
MODELING OF DISTRIBUTED MUTUAL EXCLUSION SYSTEM USING EVENT-B
}

\author{
Raghuraj Suryavanshi ${ }^{1}$ and Divakar Yadav $^{2}$ \\ ${ }^{1}$ Institute of Engineering and Technology, GBTU, Lucknow, INDIA \\ 2 Department of Computer Science, South Asian University, New Delhi 110067, India \\ suryavanshi.csedietlucknow.edu; dsyadavecs.sau.ac.in
}

\begin{abstract}
The problem of mutual exclusion arises in distributed systems whenever shared resources are concurrently accessed by several sites. For correctness, it is required that shared resource must be accessed by a single site at a time. To decide, which site execute the critical section next, each site communicate with a set of other sites. A systematic approach is essential to formulate an accurate speciation. Formal methods are mathematical techniques that provide systematic approach for building and verification of model. We have used Event-B as a formal technique for construction of our model. Event-B is event driven approach which is used to develop formal models of distributed systems. It supports generation and discharge of proof obligations arising due to consistency checking. In this paper, we outline a formal construction of model of Lamport's mutual exclusion algorithm for distributed system using Event-B. We have considered vector clock instead of using Lam-port's scalar clock for the purpose of message's time stamping.
\end{abstract}

\section{KEYWORDS}

Formal Methods, Distributed System, Vector Clock, Event-B, Formal Specifications, Mutual Exclusion.

\section{INTRODUCTION}

In distributed system, the problem of mutual exclusion arises when several sites access shared resources concurrently. To ensure the correctness, it is necessary that the shared resource must be accessed by a single site at a time. The mutual exclusion problem in a single computer system, where shared memory exist, can be solved by using shared variables i.e., semaphores. In distributed systems, shared memory does not exist and the resources may be distributed. Therefore, approaches based on shared variable may not be applicable. To solve the problem of mutual exclusion in distributed system, the approaches based on message passing are used. The mutual exclusion algorithm can be categorized as token based [1], [2] and non token based algorithm [1], [3], [4]. In the first category a unique token is shared among all the sites. A site is allowed to enter its critical section if it contains the token. In non token based algorithm, a site communicates with a set of other sites to decide who should execute the critical section next. Non token based mutual exclusion algorithms use timestamps to order requests for the critical section.

Jan Zizka (Eds) : CCSIT, SIPP, AISC, PDCTA - 2013

pp. 477-491, 2013. C CS \& IT-CSCP 2013

DOI : $10.5121 /$ csit.2013.3653 
In this paper, formal construction of non token based mutual exclusion algorithm for distributed system is outlined. We have considered Lamport's algorithm [1], [3] for formal development of our model. In this algorithm, each site maintains a request queue, which contains its own times tamped request for mutual exclusion and also request messages received from other sites [3]. If any site $S x$ wants to enter the critical section, it broadcasts a time stamped request message REQUEST-X to all the sites and makes an entry for request message REQUEST-X in its request queue. When a site $S y$ receives the request message REQUEST-X sent by site $S x$, It makes an entry of Sx's request $(R E Q U E S T-X)$ in its request queue and returns a time stamped reply message REPLY-Y to site Sx. After receiving the time stamped reply messages from all the sites, there questing site $S x$ enters the critical section if following conditions hold :

1. Time stamp of all received messages are greater than time stamp of request message REQUEST-X.

2. Time stamp of REQUEST-X is minimum among all requests present in request queue of site $S x$.

After executing the critical section site $S x$ removes the entry of request message (REQUEST-X) from its request queue and broadcasts a time stamped release message RELEASE-X to all the sites. When a site $S y$ receives the release message RELEASE- $X$ from site $S x$, It removes $S x$ 's request REQUEST-X from its request queue. When a site removes a request from its request queue then it may possible that next minimum times tamped request is own request, enabling it to enter the critical section. This algorithm executes critical section requests in the increasing order of timestamps.

A functional specification of system describes its behavior. A specification contains significant information about the system. The B Method provides a systematic approach to formulate an accurate specification. we develop our model in the spirit embedded in Event-B. The model contains a BROADCAST-REQ event that models the event for requesting critical section. In this event a requesting site broadcasts a time stamped request message to all sites. Delivery of time stamped request message is shown by DELIVER-REQ event. The event REPLY models the event for sending time stamped reply message from a site (receiver of request message) to requesting site. The event REPLY-RECEIVE models the receiving of time stamped reply message at the requesting site. At the same time this event also count how many sites have sent the reply messages. The execution and releasing of critical section is shown by the event EXECUTE-CS and RELEASE-CS respectively. After the execution of critical section, the requesting site broadcasts a timestamped release message to all sites. The broadcasting of time stamped release message is shown by the event BROADCAST-RELEASE. The event DELIVER-RELEASE models the delivery of times tamped release message at all sites.

The remainder of this paper is organized as follows: Section 2 briefly outline Event B and Rodin platform, Section 3 describes system model and informal description about events, Section 4 presents Event-B Model of mutual exclusion for distributed system. Section 5 concludes the paper 


\section{EVENT-B AND RODIN PLATFORM}

The B Method [5], [6], [7] is a model oriented state based method. It represent the complete mathematical development of a Discrete Transition System. Event-B represents a further evolution of the B method, which has been simplified and is now centered around the general notion of events. Event-B [8], [9],[10], [11], [12],[13], [14], [15], [16], [17] is event driven approach used to develop formal models of distributed systems. It is made of several components of two kinds: machines and contexts. Machines represent the dynamic part of model. This part is used to provide behavioral properties of model. It contains the variables, invariants, theorems, and events of a project. A machine is made of a state, which is defined by means of variables. Variables correspond to mathematical objects: sets, binary relations, functions, numbers, etc. These variables are constrained by invariants and these invariants are to be preserved while change the value of variables. The theorem of machine must follow from the context and the invariants of that machine. Moreover, a machine can be refined by other machines, but each machine can refine only one machine. Contexts contain the static part of model. It contains sets, constants, axioms, theorems. Sets may be enumerated or carrier. Axioms are used to describe the properties of those sets and constants. The context may be seen by machine directly or indirectly.

Besides its state, a machine contains a number of events which specify how the state may evolve. An event is made up of three elements its name, guards and actions. The guards are the necessary conditions for the event to occur. An event known as initialization event has no guard and it gives initial position of the model. An event can be specified in one of following three forms:

\section{Event $\triangleq$ any $\mathrm{k}$ where $\mathrm{P}(\mathrm{k}, \mathrm{v})$ then $\mathrm{S}(\mathrm{k}, \mathrm{v})$ end$$
\text { Event } \triangleq \text { when } \mathrm{P}(\mathrm{v}) \text { then } \mathrm{S}(\mathrm{v}) \text { end }
$$$$
\text { Event } \triangleq \text { begin } \mathrm{S}(\mathrm{v}) \text { end }
$$

Where $\mathrm{k}$ denotes parameters that are local to event, $\mathrm{v}$ denotes variable of machine containing the event, $\mathrm{P}(\ldots)$ is a predicate denoting the guards of event and $\mathrm{S}(\ldots)$ denotes the actions that updates some variables. Event-B notations are set theoretic notations. The syntax and description of notations are outlined in [10].

The Event-B Method requires the discharge of proof obligations for consistency checking. What is to be proved is stated in terms of proof obligations of a model. Proof obligations serve to verify properties of a model. They also serve to demonstrate that a model is sound with respect to some behavioral semantics. In this work, we have used Rodin platform. It is an open extensible tool for specification and verification of Event-B. The tool provides a seamless integration between modeling and proving. It also provide an environment for generation and discharge of proof obligations. It is embedded by various plugins such as proof-obligation generator, model checkers, provers, UML transformers, etc.

\section{SYSTEM MODEL}

We have considered a distributed system having a set of sites where every site maintains a request queue. The request queue contains timestamped request messages. In our model, time stamping of messages are done through vector clock [18]. In a system of vector clock, every site maintains a 
vector of size $\mathrm{N}$ to represent what that site believes to be the logical time at all other sites $(\mathrm{N}$ is the total number of sites in the system). Assume each site $S i$ maintains a vector clock $V T_{S i}$, where $V_{T_{s i}}(i)$ represents a local logical time at $S_{i}$ while $V T_{s_{i}}(j)$ represents the site $S i$ 's latest knowledge of the time at site $S j$. Precisely $V T_{S i}(j)(i \neq j)$ represents the local time at site $s_{S j}$ when the most recent message was sent from $S j$ to $S i$ directly or indirectly. Each time when a message is sent by any particular site a vector time stamp is assigned to message. While sending a message $M$ from site $S i$ to $S j$, sender process $S i$ updates its own time (ith entry of vector) by updating $V T_{S i}(i)$ as $V T S i(i):=V T_{S i}(i)+1$. The message time stamp $V T M$ of message $M$ is generated as $V T_{M}(k):=$ $V T_{s i}(k), \forall k \in(1 . . M)$, where $N$ is the number of sites in system. A site $S i$ increments its own local time $V T_{S i}(i)$ only at the time of sending a message.

When a recipient site $S j$ receives a time stamped message it updates its knowledge by updating own vector clock. Site $S j$ updates its vector clock $V T_{S j}$ afterthe delivery of message $M$ as $V T_{S j}(k)$ $:=\operatorname{Max}(\operatorname{VTSj}(k), V T M(k))$. Therefore, inthe vector clock of site $S j, V T_{S j}(i)$ indicates the number of messages delivered to site $S j$ sent by site $S i$. The delivery order of messages between every pair of sites must follow FIFO order. The FIFO ordering property says that: If a particular site broadcasts a message $M 1$ before it broadcast a message M2, then each recipient process delivers M1 before M2. The informal description of events are as follows:

1. Request for Critical Section: Any site which wants to enters the critical section, broadcasts a time stamped request message to all the sites. When a site broadcasts a message it increments its own vector time stamp by one and modified vector time stamp is assigned to message. It also creates an entry of time stamped request message in its request queue.

2. Delivery of Request Message: When a site receives the time stamped request message, it makes an entry of received request in its request queue. The delivery order of request message must follow the FIFO order. This ensures that all the messages which are previously sent by requesting site before the request message have already been delivered. During the delivery of request message receiving site also updates its knowledge by updating own vector time stamp with the time stamp of request message.

3. Reply to Requesting Site: After the delivery of timestamped request message at any site, it sends a corresponding time stamped reply message to requesting site. For assigning time stamp to message, a receiving site increments its own vector time stamp by one and modified vector time stamp is assigned to reply message.

4. Receive Reply Message: The requesting site receives the times tamped reply message sent by all sites. It makes an entry for each received reply message.The requesting site also count the total number of replied site. Each time when it receives a reply message it increments the value of total number of replied site by one. The requesting site also updates its vector time stamp with the time stamp of replied messages.

5. Execution of Critical Section: After receiving the reply messages from all sites, a requesting site enters critical section if the time stamp of all received messages are greater than time stamp of its request message and also the time stamp of own request message is minimum among all request messages present in request queue. 
6. Release Critical Section: After performing execution of critical section the requesting site release it and removes the entry of request message from its request queue.

7. Broadcast Release Message: The requesting site broadcasts a time stamped release message to all the sites so that they can also remove the entry of request message (which is previously sent by it) from their request queue.

8. Receive Release Message: After the delivery of timestamped release message at any site, it removes the entry of corresponding request message from its request queue and updates its vector time stamp with the time stamp of release message.

\section{EVENT-B MODEL OF MUTUAL EXClusion FOR DistribUted SYSTEM}

Our Event-B model contains a context and a machine having eight events. In a context seen by machine SITE and MESSAGE represent carrier set. The status is defined as enumerated set containing the element pending, reqcs, execs, releasecs. The type is also defined as enumerated set and contains the element request, reply, release. Variable sender is defined as a partial function from MESSAGE to SITE. A mapping of the form $(m 7 \rightarrow s) \in$ sender indicates that message $m$ was sent by a site $s$. The variable msgsend is subset of MESSAGE and it contains only those messages which are sent by any site. The variable reqsites is subset of SITE and it contains only those sites which have sent request messages. The variable vtss represents vector time stamp of site. It is declared as:

$$
v t s s \in \text { SITE } \rightarrow(\text { SITE } \rightarrow \text { Natural })
$$

It is a total function which maps every site to a vector function. The vector function maps each site to a natural number. The 'Natural' represents a set of natural numbers in B. Therefore, vector time stamp of any site $S i$, vtss $(\mathrm{Si})$ is a vector. The length of vector depends on number of sites present in the set SITE. Assume there are $K$ sites in the system then $v t s s(\mathrm{Si})$ is a vector of $((S 17 \rightarrow N 1),(S 27 \rightarrow N 2),(S 37 \rightarrow N 3) \ldots \ldots \ldots(S i 7 \rightarrow N i) \ldots \ldots . .(S k 7 \rightarrow N k))$.

Every time when a message is sent by site $S i$, it increments its own clock value vtss $(\mathrm{Si})(\mathrm{Si})$ by one. Therefore, vector time stamp of site $S i$ after sending single message is

$((S 17 \rightarrow N 1),(S 27 \rightarrow N 2),(S 37 \rightarrow N 3) \ldots \ldots . .(S i 7 \rightarrow N i+1) \ldots \ldots .(S k 7 \rightarrow N k))$.

The variable $v t s m$ represents vector time stamp of message. It is defined as: 


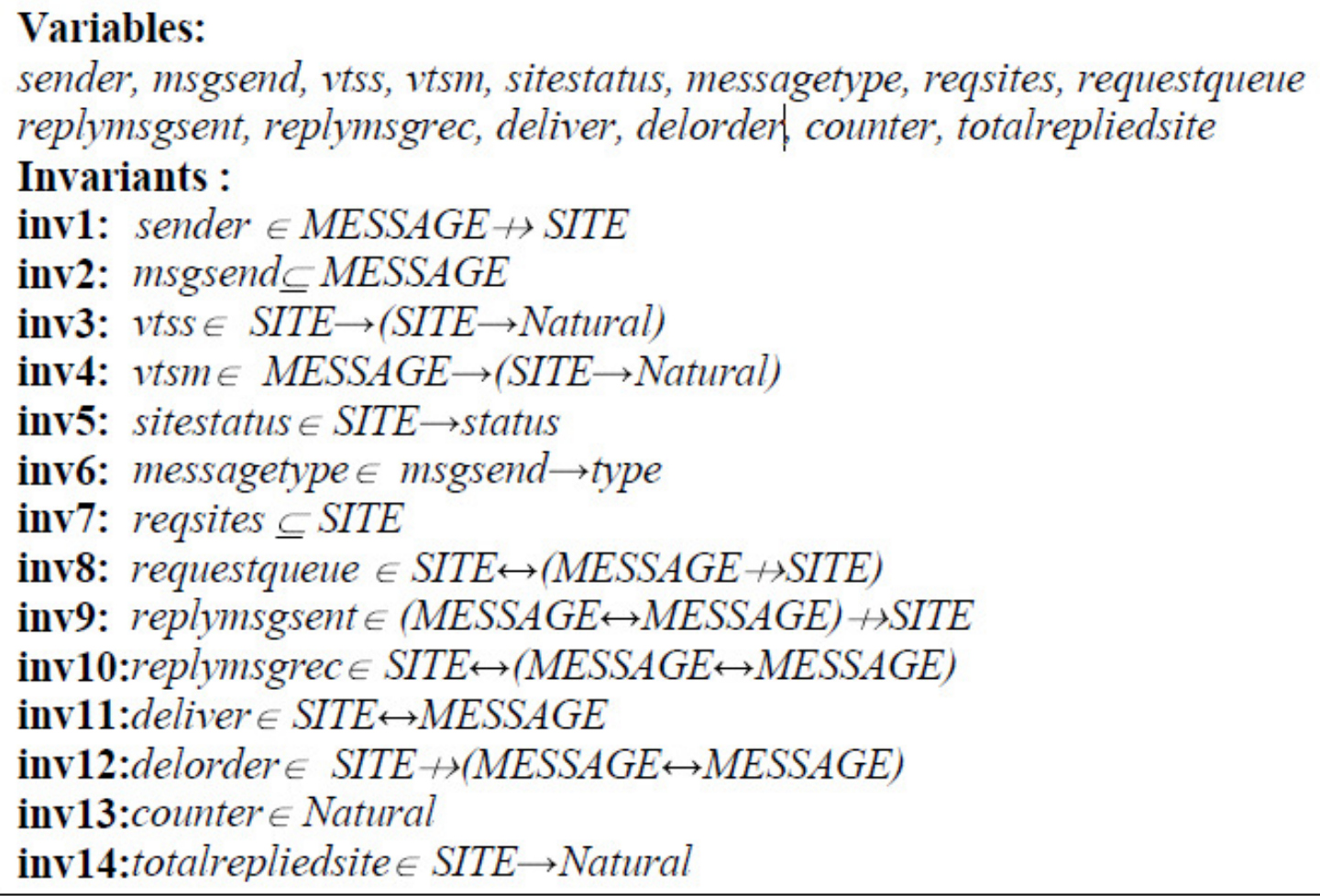

Fig. 1. Variables and Invariants of Machine

$$
v t s m \in \text { MESSAGE } \rightarrow(\text { SITE } \rightarrow \text { Natural })
$$

It is a total function which maps every message to a vector function. Vector time stamp of any message $\mathrm{mm}(\mathrm{vtsm}(\mathrm{mm}))$ is also a vector. Every time when a message $\mathrm{mm}$ is sent by site $\mathrm{Si}$, it increments its own clock value by one and modified vector timestamp of site is assigned to message $\mathrm{mm}$. This creates thevector timestamp of message $\mathrm{mm}$. The $v t s s(\mathrm{Si})(\mathrm{Si})$ represents the number of messages sent by site Si. The description of other variables are as follows (see Fig. 1):

(i) The variable sitestatus is defined as a total function which maps each site to status. Thus every site in the set SITE will have one of the following states;pending, reqcs, execs, releasecs.

(ii) The variable messagetype is defined as:

messagetype $\in$ msgsend $\rightarrow$ type

It is a total function which maps every sent message to type. This ensures that every sent message will have one of the following states; request, reply, release.

(iii) The variable requestqueue is declared as:

requestqueue $\in$ SITE $\leftrightarrow(M E S S A G E \rightarrow S I T E)$

The operator $\leftrightarrow$ defines the set of relations between SITE and request messages sent by corresponding sites. A mapping of the form $(s s \mapsto(m \mapsto s)) \in$ 


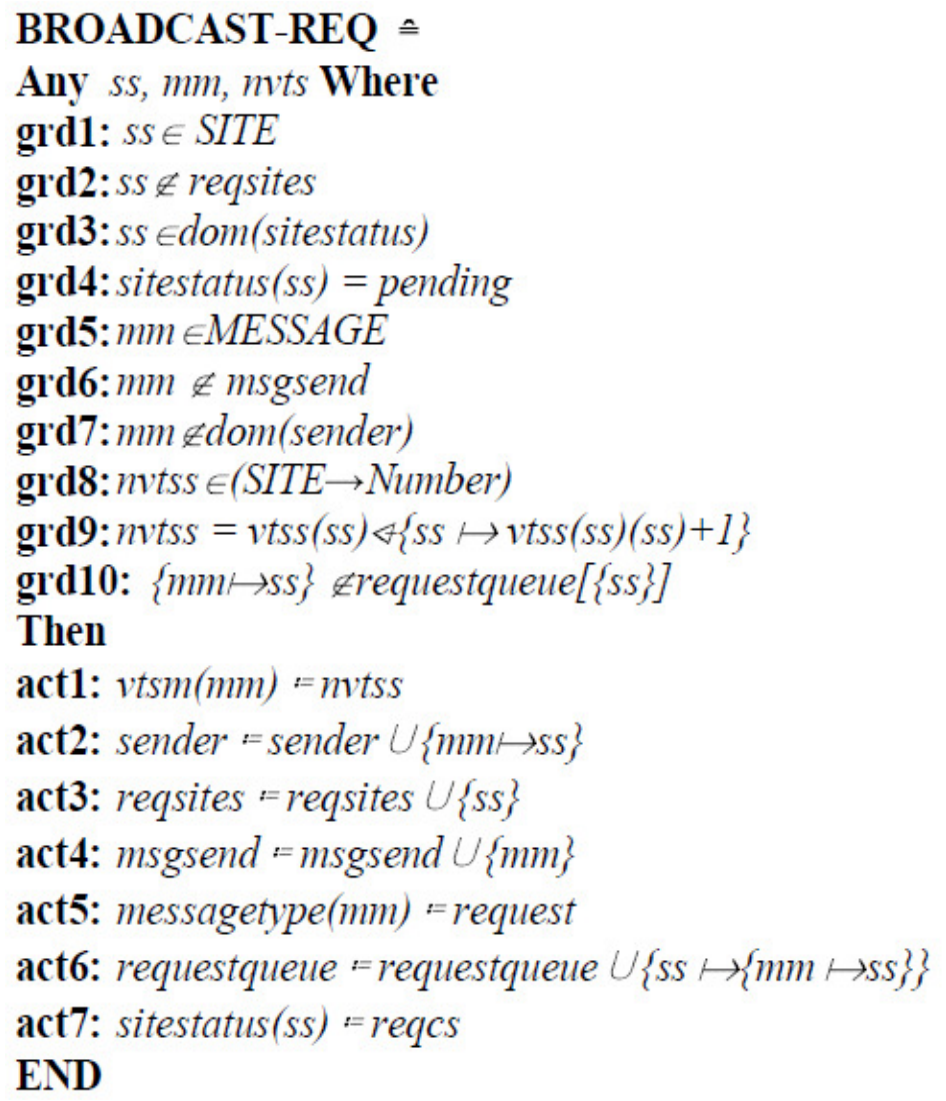

Fig. 2. Broadcasting of request message

requestqueue indicates that request queue of site $s s$ has a request message $m$ sent by site $s$. Relational image of site $\mathrm{Si}$ under the relation requestquеие is represented by requestqueиe[\{Si\}] and it contains all request messages sent by corresponding sites i.e., if site $S i$ receives three request messages $M 1, M 2, M 3$ sent by sites $S 1, S 2, S 3$ respectively then requestqueue[\{Si\}] contains $\left(\left(M_{1} \mapsto S_{1}\right),\left(M_{2} \mapsto S_{2}\right),\left(M_{3} \mapsto S_{3}\right)\right)$. The vector time stamp of messages can be found from the variable vtsm.

(iv) When a site receives a request message it sends a corresponding reply message to requesting site. A reply of request message sent by a site is represented by variable replymsgsent. It is defined as:

$$
\text { replymsgsent } \in(\text { MESSAGE } \leftrightarrow \text { MESSAGE }) \rightarrow \text { SITE }
$$

A mapping $\{(\{m m \mapsto m\}) \mapsto s s\} \in$ replymsgsent indicates that a reply message $m$ of a request message $m m$ has been sent by a site ss.

(v) The variable replymsgrec represents receiving of reply message of a request message at requesting site.

(vi) The variable deliver represents delivery of message at a site. A mapping of form $(s \mapsto m) \in$ deliver deliver represents that a site $s$ has delivered message $m$. 


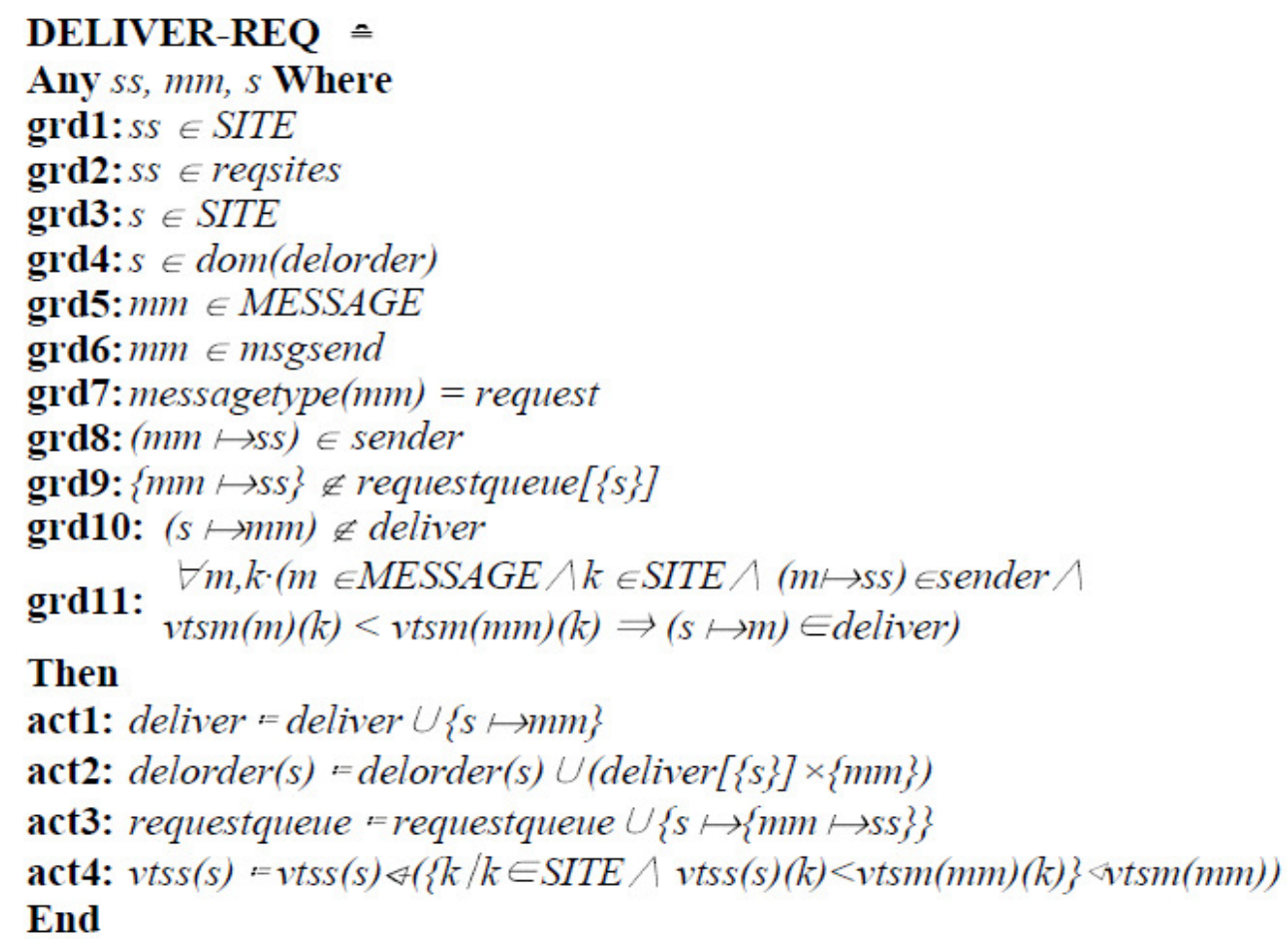

Fig. 3. Delivery of request message

(vii) The variable delorder represents delivery order of messages at a site. A mapping $(m 1 \mapsto m 2) \in \operatorname{delorder}(s)$ indicate that site $s$ has delivered $m 1$ before $m 2$.

(viii) The variable totalrepliedsite maps each site to 'Natural' number. Variable counter is a integer type which is used to count number of sites from which requesting site has received the reply messages. A mapping $(s \mapsto n) \in$ totalrepliedsite represents that ' $n$ ' number of sites has sent reply message to site $s$. Each time when a requesting site receives a reply message from other sites the value of counter is incremented by one. Initially, the status of all site is set to as pending and the value of variable counter is zero. The vector time stamp of all sites and messages are initialized with zero. The remaining variables contain null values.

Broadcasting and Delivery of Request Message : The event BROADCAST-REQ models the broadcasting of request message (see Fig. 2). A site ss which wants to enters critical section, broadcasts a timestamped request message $\mathrm{mm}$ to all site. The guard $\operatorname{grd} 6 \& \operatorname{grd} 7$ ensures that message $\mathrm{mm}$ has not been sent previously. At the time of broadcasting a message $\mathrm{mm}$, site ss increments its own clock value $v t s s(s s)(s s)$ by one $(\operatorname{grd} d 9)$. The modified vector timestamp of site is assigned to message $m m(a c t 1)$. The guard $\operatorname{grd} 10$ is written as: 


\section{REPLY $\cong$}

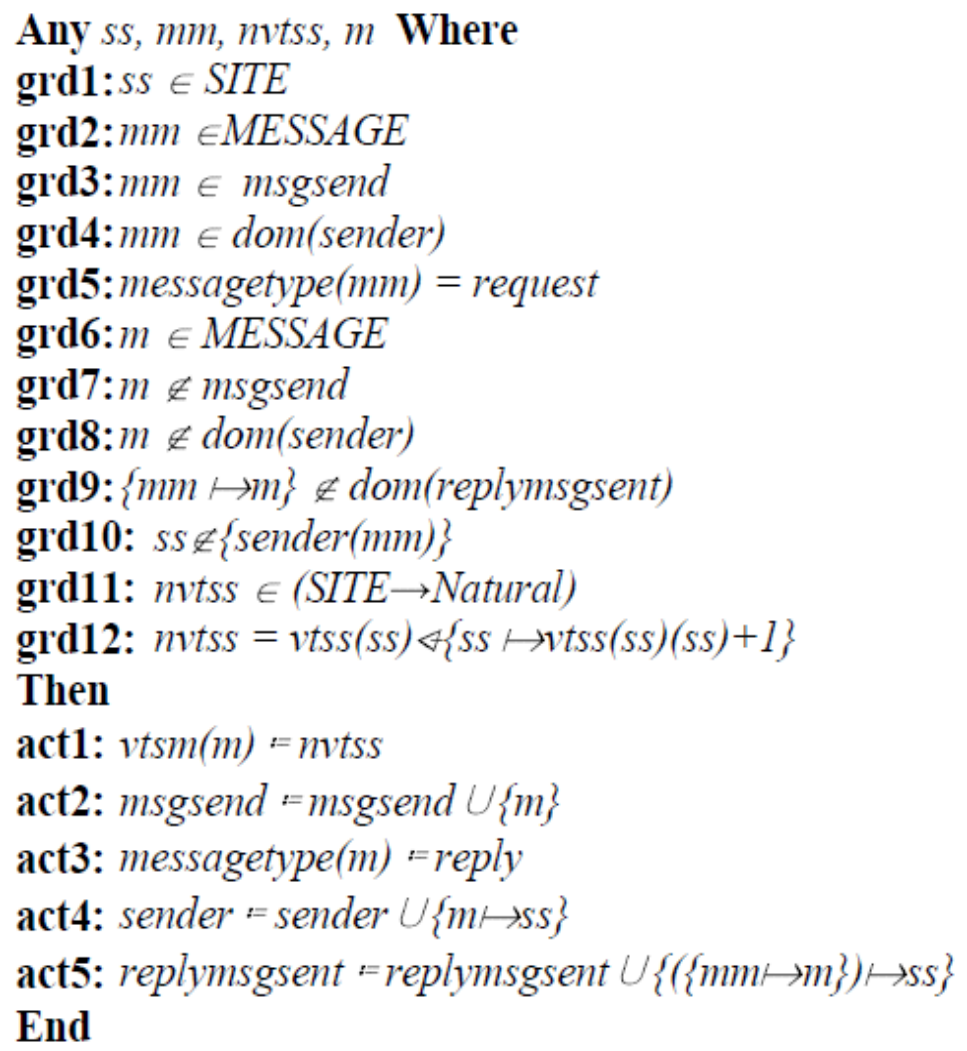

Fig. 4. Sending of Reply Message

$$
\{m m \mapsto s s\} \notin \text { requestqueue }[\{s s\}]
$$

It ensures that request queue of site $s s$ does not contain a request message $\mathrm{mm}$ which is sent by it. The action act 2 ensures broadcasting of message $m m$ by site $s s$ and actions act3, act 4 add the site ss and message $\mathrm{mm}$ in the set reqsites and msgsend repectively. The type of message $\mathrm{mm}$ is set to as a request through the action act5. The action act6 adds the message $\mathrm{mm}$ sent by site $\mathrm{ss}$ in the request queue of ss. The action act 7 changes the status of site ss from pending to reqcs.

The event DELIVER-REQ models the delivery of request message (see Fig.3).The request message $\mathrm{mm}(\mathrm{grd})$ which is sent by site $s s(\mathrm{grd})$ has not been delivered at site $s$ is ensured by guard $\operatorname{grd} 10$. The site $s s$ is requesting site is ensured by guard $\operatorname{grd} 2$. The guard $\operatorname{grd} 9$ ensures that request message $\mathrm{mm}$ sent by site $s s$ is not present in the request queue of site $s$. The guard grd11 ensures FIFO order delivery of message. It confirms that all the messages which are sent by site $s s$ before message $\mathrm{mm}$ has been already delivered to site $s$. As a consequence of occurrence of this event delivery of message $\mathrm{mm}$ is done at sites (act1) and request is added in the request queue of site $s$ (act3). The delivery order at site $s$ is also updated such that all messages delivered at site $s$ must 


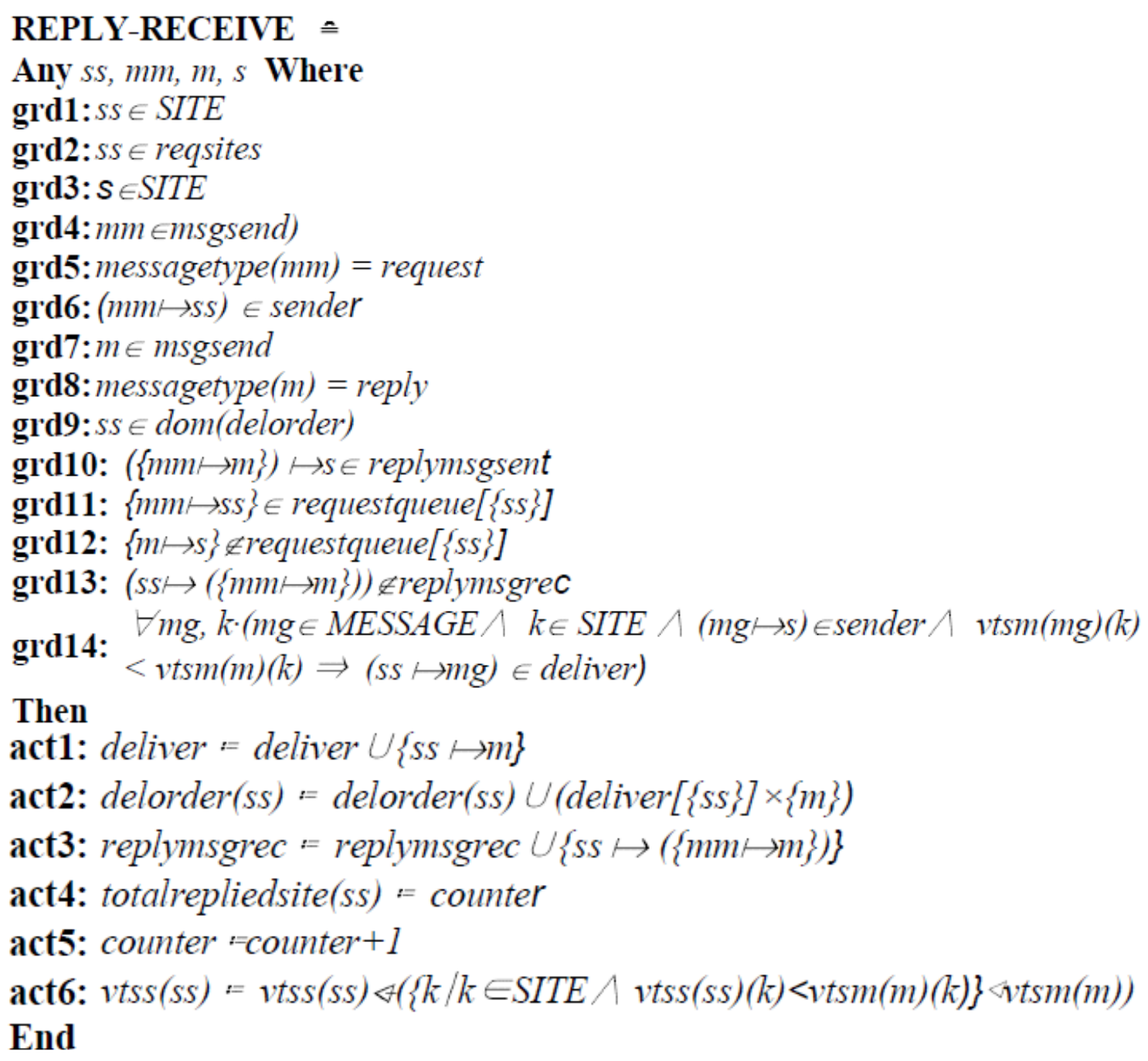

Fig. 5. Delivery of Reply Message

precede $m m$ (act2). For maintaining the latest knowledge about the system, site $s$ updates its vector time stamp. It is expressed as act4 :

$$
\operatorname{vtss}(\mathrm{s}):=\operatorname{vtss}(\mathrm{s}) \nLeftarrow(\{\mathrm{k}: \mid \mathrm{k} \in \operatorname{SITE} \wedge \operatorname{vtss}(\mathrm{s})(\mathrm{k})<\operatorname{vtsm}(\mathrm{mm})(\mathrm{k})\} \triangleleft \operatorname{vtsm}(\mathrm{mm}))
$$

The operator $\&$ (overload operator) updates the values in the vector clock of site $s$ by corresponding values in the vector timestamp of message $m m(v t s m(m m))$ wherever values in the recipient site clock $(v t s s(s)(k))$ are less than corresponding values in the message time stamp $(v t s m(m m)(k))$.

Sending and Delivery of Reply Message: The event REPLY is given in Fig.4. This event models the sending of timestamped reply message of corresponding request message. The message $\mathrm{mm}$ is request message is ensured by guard grd5. A reply message $m$ of request message $\mathrm{mm}$ has not 
been sent is ensured by guards $\operatorname{grd} 7 \& \operatorname{grd} 9$. The guard $\operatorname{grd} 8$ ensures that message $m$ is a fresh message and has not been previously sent by any site. As a consequences of occurrence of this event, incremented vector time stamp value of site $s s$ is assigned to message $m$ (actl) and it is added in the set msgsend (act2). The type of message $m$ is set to as reply through action act 3 . The action act4 makes site $s s$ as a sender of

$m$. The action $a c t 5$ is written as:

$$
\text { replymsgsent }:=\text { replymsgsent } \cup\{(\{m m \mapsto m\}) \mapsto s s\}
$$

It updates variable replymsgsent and creates the entry of reply message $m$ of request message $m m$ sent by site ss. The event REPLY-RECEIVE models the delivery of reply message at requesting site (see Fig. 5). Site ss is a requesting site is ensured by guard grd2.

A request message $m m$ has already been sent by site $s s$ is ensured by guards $g r d 4, \operatorname{grd} 5 \& \operatorname{grd} 6$. A reply mesaage $m$ of $\mathrm{mm}$ has been sent by site $s$ is ensured by guard grdlo. The guard $\mathrm{grdl} 3$ ensures that reply $m$ of corresponding request message $m m$ has not been received by site ss. The guard grd14 ensures FIFO order delivery of message. The action actl makes the delivery of message $m$ at sites $s s$ and action act 2 updates the delivery order of messages such that all messages delivered at site $s s$ must precede $m$. The action act 3 is written as:

$$
\text { replymsgrec }:=\text { replymsgrec } \cup\{\text { ss } \mapsto(\{m m \mapsto m\})\}
$$

This makes receiving of reply message $m$ of request message $m m$ at site $s s$. This event also count how many sites have sent the reply message to requesting site. Each time when a reply message is received by requesting site the value of total replied site is incremented by one (act $4 \&$ act5). For maintaining the latest knowledge about the system, site ss updates its vector time stamp through the action act6.

Execution and Releasing of Critical Section: The EXECUTE-CS event, given in Fig. 6, models the execution of critical section. A requesting site ss executes the critical section if following condition holds:

(i) Site $s s$ has received the reply messages from all sites and time stamp of all received messages are greater than time stamp of request message which is sent by site ss.

(ii) Time stamp of all request messages which are present in the request queue of site ss are greater than the time stamp of request message sent by site $s s$. The guard grd2 ensures that site $s s$ is requesting site and guard grd4 ensures that status of site $s s$ is reqcs. The message type of $\mathrm{mm}$ is request is ensured by guard $g r d 7$. The guard $\operatorname{grd} 8$ ensures that request queue of site $s s$ contains a request message $\mathrm{mm}$ which is sent by it. The guard $g r d 9$ ensures that site $s s$ has received the reply messages from all the sites. The guard grdlo ensures that time stamp of request message $\mathrm{mm}$ is less than time stamp of all received replied messages. The guard grd11 ensures that time stamp of request message $\mathrm{mm}$ is minimum among all the requests messages present in request queue of site ss. The status of site ss is set to as execs through the action actl. 


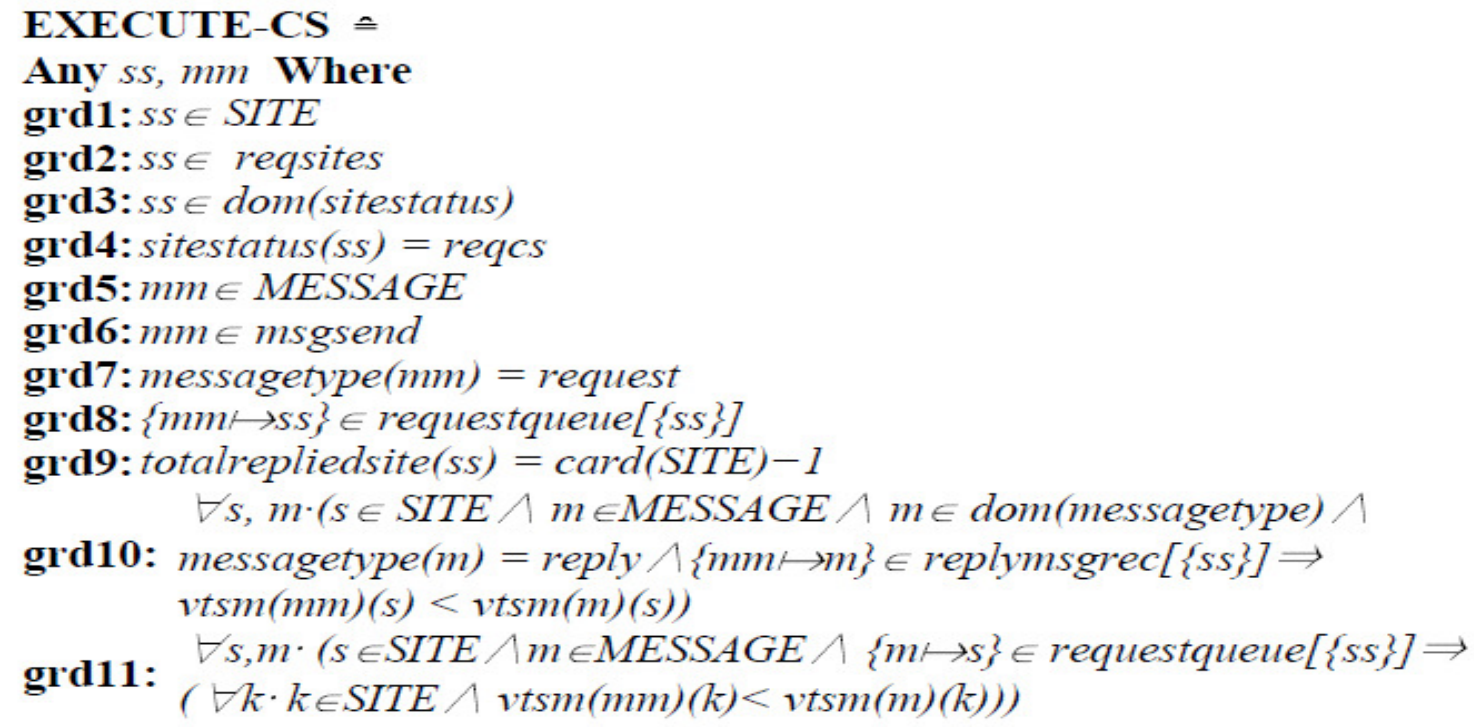

Then

act1: sitestatus $($ ss $):=$ execs

End

RELEASE-CS $=$

Any $s s, m m$ Where

grd1: $s s \in$ SITE

grd2: $s \in$ reqsites

grd3: $s s \in$ dom(sitestatus)

grd4: $\operatorname{sitestatus(ss)}=$ execs

grd5: $m m \in M E S S A G E$

grd6: $m m \in$ msgsend

grd7: messagetype $(\mathrm{mm})=$ request

grd8: $\{m m \mapsto s s\} \in$ requestqueue $[\{s s\}]$

Then

act1: requestquene $:=$ requestquene $\backslash\{\mathrm{ss} \mapsto\{m m \mapsto s \mathrm{~s}\}\}$

act2: sitestatus(ss) $:=$ releasecs

End

Fig. 6. Execution and Releasing of Critical Section

The RELEASE-CS event models the releasing of critical section (see Fig.6).After performing execution of critical section the requesting site release it and removes the entry of request message from its request queue. The site $s s$ is requesting site is ensured by guard grd2. The guard grd4 ensures that status of site $s s$ is execs. This event set the status of site $s s$ as releasecs (act2) and removes entry of its request from its request queue (actl). 


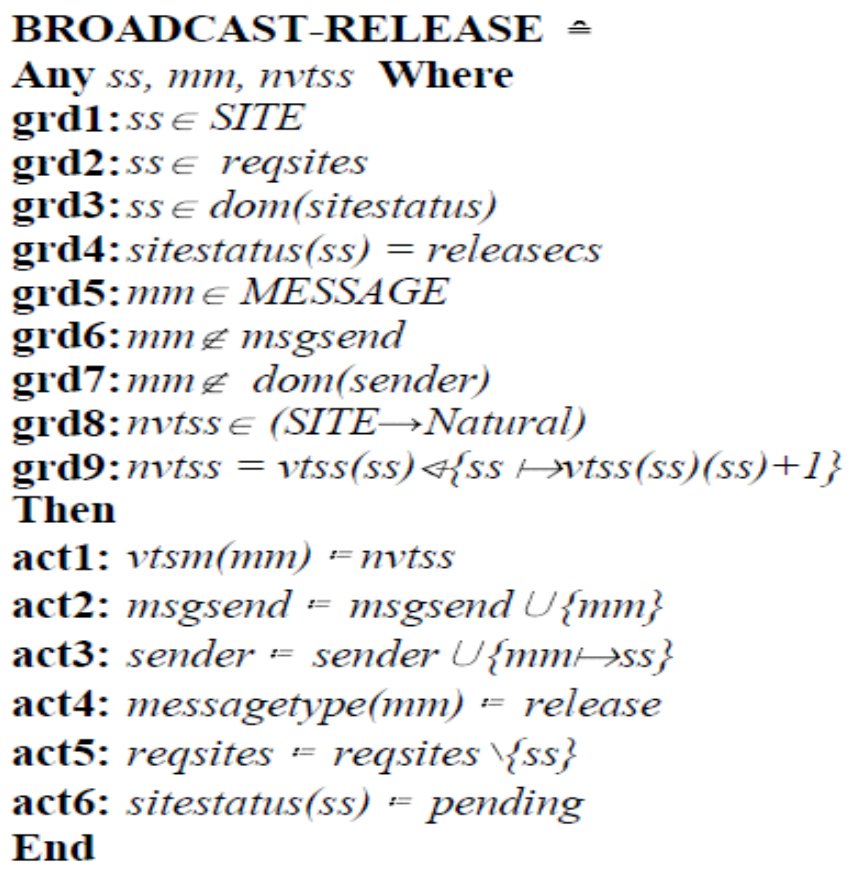

Fig. 7. Broadcasting of Release message

Broadcast and Delivery of Release message: The event BROADCAST-RELEASE is given in Fig. 7. After releasing of critical section site ss broadcasts a time stamped release message to all sites so that they can also remove the entry of request message previously sent by it. The guard grd4 ensures that site ss has performed the execution of critical section. Before broadcasting a reply message $\mathrm{mm}$ site $s s$ increments its vector time stamp ( $\mathrm{grd} 9$ ) and this modified vector time stamp is assigned to the message $\mathrm{mm}$ (actl). The action act4 set the type of message $\mathrm{mm}$ as release message. The action act 5 removes site $s s$ from request set reqcs. The status of site $s s$ is set to as pending through the action (act6).

The event DELIVER-RELEASE models the delivery of release message (see Fig. 8). The guard grd3 ensures that message $\mathrm{mm}$ is release message. A site $s s$ has sent the release message $\mathrm{mm}$ is ensured by guard $\operatorname{grd} 4$. In the request queue of site $s$ (recipient site) there is an entry of request message $m$ sent by site $s s$ is ensured by guard $\operatorname{grd} 9$. The guard $\operatorname{grd} 10$ ensures FIFO order delivery of messages. The delivery of message $\mathrm{mm}$ at site $s$ is done through action actl. The action act 2 updates the delivery order such that all the messages which are previously delivered to site $s$ must precede message $\mathrm{mm}$. The action act3 updates the vector time stamp of site $s$. The action act4 removes the entry of request message $m$ sent by site $s s$ from the request queue of site $s$. Removing a request from request queue makes possible that next minimum time stamped request is own request, enabling it to enter the critical section. 


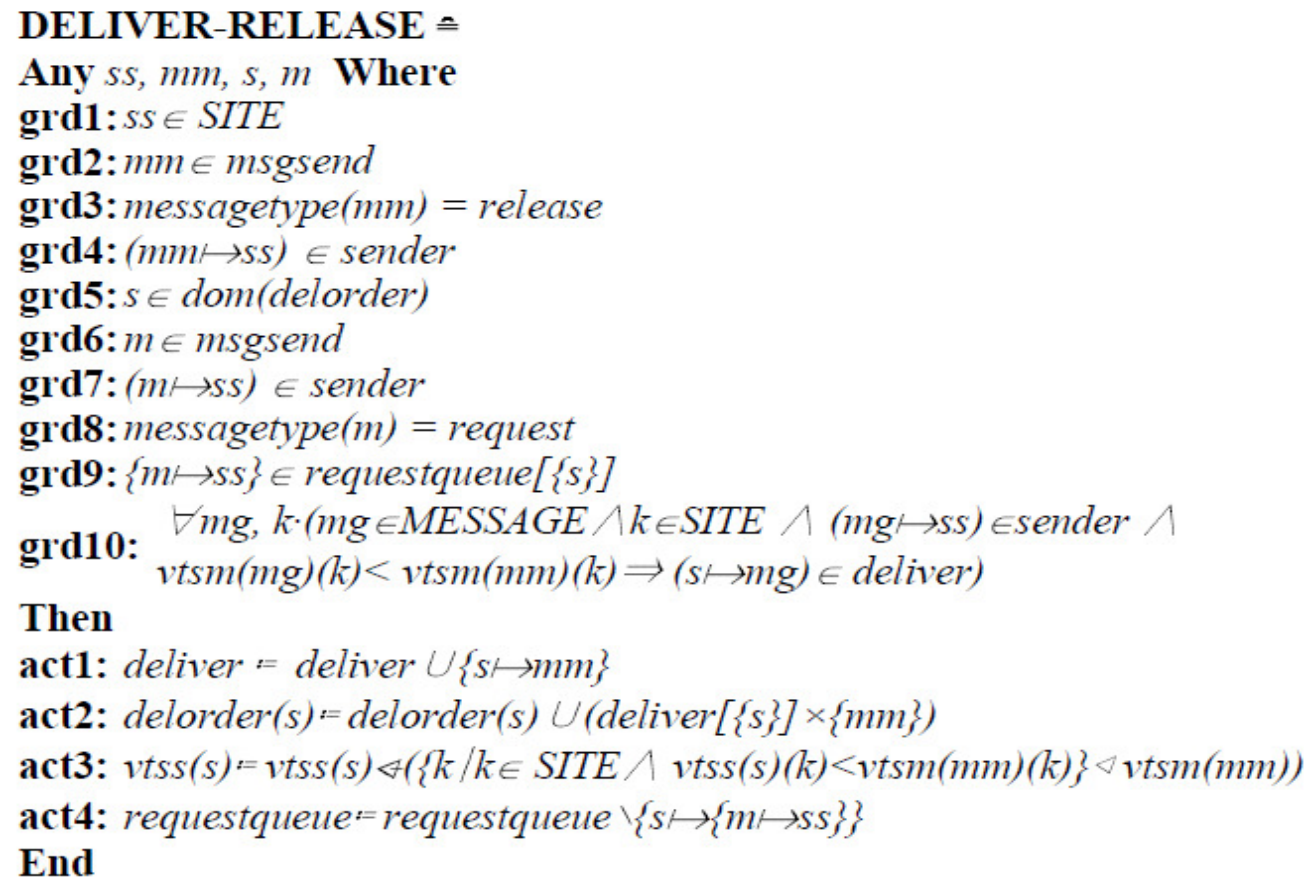

Fig. 8. Delivery of Release message

\section{CONCLUSIONS}

In distributed system, due to absence of global clock and shared memory traditional technique like semaphore may not be appropriate for solving the problem of mutual exclusion. To decide which site execute the critical section, a site communicates with other sites by sending a message. We have considered Lamport's mutual exclusion algorithm [1], [3] for formal construction of our model. In this algorithm, each site maintains a request queue, which contains its own timestamped request for mutual exclusion and also request messages received from other sites [3]. We have considered vector clock [18] instead of using Lamport's scalar clock for assigning the time stamp to messages. In a system of vector clock, every site maintains a vector to represent what that site believes to be the logical time at all other sites.

In this paper, modeling of distributed mutual exclusion system is specied using Event-B. This work is carried out on Rodin tool [16], [17]. The Rodin tool is intended to support construction and verification of Event-B models. The tool takes the formal text of model and produces proof obligations. It provides an environment to discharge of proof obligations arising due to consistency checking. Modeling guidelines outlined in [14] were used and these guidelines helped us in modeling and discharging proof obligations generated due to consistency checking. Total sixty four proof obligations were generated by the system and all of them were discharged automatically. The proofs and invariants together helped us to reason about the system design.We also found that vector clock can also be used to implement Lamport's mutual exclusion instead of using scalar clock. 


\section{REFERENCES}

[1] Singhal, M., Shivratri, N.G.: Advanced Concepts in Operating Systems. Tata Mc- GrawHill Book Company, India (2005)

[2] Raymond, K.: A Tree-Based algorithm for Distributed Mutual Exclusion. In: ACM transactions on computer systems, vol.7, pp. 61-77, (1989)

[3] Lamport, L.: Time, Clocks and Ordering of Events in Distributed Systems. In:communications of the ACM, July (1978)

[4] Ricart, G. and Agrawala, A. K.: An Optimal algorithm for Mutual Exclusion in Computer Networks. In: communications of the ACM, (1981)

[5] Butler, M.: An Approach to Design of Distributed Systems with B AMN. In: Proc. 10th Int. Conf. of Z Users: The Z Formal Speci_cation Notation (ZUM), LNCS1212, pp. 223-241, (1997).

[6] Butler M. and Walden, M.: Distributed System Development in B. In: Proc. of Ist Conf. in B Method, Nantes, pp. 155-168, (1996).

[7] Rezazadeh, A. and Butler, M.: Some Guidelines for formal development of web based application in B Method. In: Proc. of 4th Intl. Conf. of B and Z users, Guildford, LNCS, Springer, pp 472-491, (2005).

[8] Banach, R.: Retrenchment for Event-B: UseCase-wise development and Rodin integration. Formal Aspects of Computing, 23, pp. 113131, (2011).

[9] Hallerstede, S.: On the purpose of Event-B proof obligations. Formal Aspects of Computing, 23: pp. 133150, (2011).

[10] Yadav, D. and Butler, M.: Rigorous Design of Fault-Tolerant Transactions for Replicated Database Systems Using Event B. In: Butler M., Jones, C.B.(eds.) LNCS, vol. 4157, Springer, Heidelberg, pp.343-363,(2006).

[11] Hallerstede, S. and Leuschel, M.: Experiments in program veri_cation using Event-B. Formal Aspects of Computing, 24: pp. 97125, (2012)

[12] Suryavanshi, R. and Yadav, D.:Formal Development of Byzantine Immune Total Order Broadcast System using Event-B. In: ICDEM 2010, Andres, F. and Kannan, R. (eds.) LNCS, Vol. 6411, Springer, pp.317-324, (2010).

[13] Yadav, D. and Butler, M.: Application of Event B to Global Causal Ordering for Fault Tolerant Transactions. In: Proc. of REFT 2005, Newcastle upon Tyne, pp. 93-103, (2005).

[14] Butler, M. and Yadav, D.: An incremental development of the mondex system in Event-B. Formal Aspects of Computing, 20(1):61-77, (2008).

[15] Yadav, D. and Butler, M.: Formal Development of a Total Order Broadcast for Distributed Transactions Using Event-B. Lecture Notes in Computer Science 5454, springer-Verlag Berlin Heidelberg, pp.152-176, (2009).

[16] Metayer, C., Abrial, J.R. and Voison, L.: Event-B language. RODIN deliverables 3.2, http://rodin.cs.ncl.ac.uk/deliverables/D7.pdf, (2005).

[17] Abrial, J.R.: A system development process with Event-B and the Rodin platform. In: Lecture Notes In Computer Science 4789, Springer, pp.1-3, (2007).

[18] Baldoni, R. and Raynal, M.: Fundamentals of Distributed Computing: A Practical Tour of Vector Clock Systems. In: IEEE Distributed Systems Online, Vol. 3, no. 2,(2002) 